\title{
The Effect of Accuracy Instructions on Coronavirus-Related Belief Change Following Conversational Interactions
}

\author{
Madalina Vlasceanu ${ }^{1,2,}$, Alin Coman ${ }^{1,3,}$ \\ ${ }^{1}$ Princeton University, Department of Psychology, Princeton, 08544, US \\ 2 Princeton Neuroscience Institute, Princeton, 08544, US \\ ${ }^{3}$ Princeton School of Public and International Affairs, Princeton, 08544, US \\ *mov@princeton.edu
}

\begin{abstract}
In a high-risk environment, such as during an epidemic, people are exposed to a large amount of information, both accurate and inaccurate. Following exposure, they typically discuss the information with each other. Here, we assess the effects of such conversations on beliefs. A sample of $126 \mathrm{M}$-Turk participants rated the accuracy of a set of COVID-19 statements (pre-test). They were then paired and asked to discuss these statements (low epistemic condition) or to discuss only the statements they thought were accurate (high epistemic condition). Finally, they rated the accuracy of the initial statements again (posttest). We do not find an effect of the epistemic condition on belief change. However, we find that individuals are sensitive to their conversational partners and change their beliefs according to their partners' conveyed beliefs. This influence is strongest for initially moderately held beliefs. In exploratory analyses, we find that COVID-19 knowledge is predicted by trusting Doctor Fauci, not trusting President Trump, and feeling threatened by COVID-19, whereas believing COVID-19 conspiracies is predicted by trusting President Trump, not trusting Doctor Fauci, news media consumption, social media usage, and political orientation. Finally, we find that news media consumption positively predicts believing COVID-19 conspiracies, even when controlling for demographic variables including political ideology, and that this effect is not driven by a particular news network, but instead it is a general effect of news media consumption.
\end{abstract}

Keywords: belief change; conversational interactions; COVID-19; misinformation; 


\section{Declarations}

Funding: This work was funded by the National Science Foundation [award 2027225].

Conflicts of interest: The authors declare no conflicts of interest.

Ethics approval: This work has been approved by the Princeton University IRB.

Availability of data and materials: All data and materials can be found on our OSF

page here: https://osf.io/sk4dt/

Code availability: The data analysis (in python and R) can be viewed as a jupyter

notebook here: https:/github.com/mvlasceanu/coviddyad 


\section{Introduction}

With the rise of globalization, infectious diseases have proven more and more farreaching (Saker, Lee, Cannito, Gilmore, \& Campbell-Lendrum, 2004). There is hardly a year without the emergence of a highly threatening pandemic, from H1N1 (swine flu) in 2009, to Ebola in 2014, to the Zika virus in 2017, and to COVID-19 in 2020. Fighting an epidemic involves not only developing effective treatments and ensuring wide distribution, but also informing the public of the symptoms, protective measures, and treatments associated with the disease. It becomes critically important, thus, to understand how information is acquired and incorporated into people's belief systems, and how these belief systems change after interacting with one another (Brandt \& Sleegers, 2021).

Prior work shows that when individuals learn about an epidemic, they engage in behaviors aimed at acquiring information, such as turning to news and social media for relevant content (Saker et al., 2004; Frenkel, Alba, \& Zhong, 2020). Subsequently, after being exposed to such large amounts of crisis-relevant information, people typically discuss the acquired information with each other (Liu, Jin, \& Austin, 2013). The communicative act of discussing information has been shown to influence people's memory of the studied information (Cuc, Koppel, \& Hirst, 2007; Hirst, \& Echterhoff, 2012), which in turn was found to impact the believability of information (Hasher, Goldstein, \& Toppino, 1977; Fazio, Brashier, Payne, \& Marsh, 2015; Vlasceanu \& 
Coman, 2018). Given that these communicative interactions shape what people believe, one strategy to diminish the believability of misinformation and to encourage the dissemination of accurate information, might be to impose a higher threshold for both communicating and accepting information. This higher threshold could be created through instructions involving high epistemic accuracy, such as encouraging people to question the veracity of information before communicating (Lewandowsky, Ecker, Seifert, Schwarz, \& Cook, 2012) or sharing (Pennycook et al., 2021) information. Prior studies, however, typically involve individual-level paradigms. Here, we assess the effectiveness of deploying this epistemic strategy on free-flowing communicative interactions, and, in turn, the impact of these interactions on people's beliefs.

Moreover, given that public health emergencies are high risk, uncertain situations that create a state of increased anxiety, they might facilitate inaccurate information to spread, mainly because people do not have the cognitive resources to assess the veracity of the information they receive (Coman \& Berry, 2015). A large body of psychological research shows that information is differentially processed by the cognitive system depending on the emotional state of the recipients (Rozin \& Royzman, 2001). Contexts high in emotionality result in high information propagation rates (Harber, \& Cohen, 2005), in "viral" successes (Berger \& Milkman, 2012), and in communicative advantages in dyadic interactions (Nyhof \& Barrett, 2001). Relatedly, uncertainty and loss of control have been found to facilitate the emergence of 
conspiracy theories (Uscinski \& Parent, 2014; Nefes, 2014; Whitson \& Galinsky, 2008; but see: Stojanov, Bering, \& Halberstadt, 2020). These findings showcase the impact of emotional states created by high-risk and high-uncertainty contexts on information propagation. Thus, in the current study we are incorporating this factor by conducting the experiment during the COVID-19 pandemic, and focusing on COVID-19 information acquisition and transfer.

To investigate the effects of conversational interactions on people's beliefs during a high-risk high-uncertainty environment caused by a global health crisis, we designed an experiment in which participants first rated the accuracy of a set of statements about COVID-19 (accurate, inaccurate, and conspiracies) and filled out a series of emotion scales. Then, they were assigned to pairs, and were asked to discuss the statements with each other, in 5-minute dyadic conversations. To manipulate the likelihood of individuals sharing accurate information in their conversational interactions, the instructions encouraged a random subset of the pairs to discuss any piece of information from the study (low epistemic accuracy condition), and the other subset of the pairs to discuss only the pieces of information they were confident were correct (high epistemic accuracy condition). Lastly, participants rated again the believability of the initial statements and their emotions.

Our first hypothesis was that participants in the high epistemic accuracy condition would become more knowledgeable than those in the low epistemic accuracy 
condition, given that the focus of their conversations would be on the accurate rather than on the inaccurate information or conspiracies. Our second hypothesis was that participants would be sensitive to their conversational partners' beliefs expressed during their conversations and adjust their own beliefs accordingly. Based on previous research (Vlasceanu \& Coman, 2018) we also hypothesized this adjustment would be strongest in the case of initially moderately held beliefs. In exploratory analyses for which we did not have a priori hypotheses, we tested the predictors of COVID-19 knowledge and conspiracy beliefs in models that included trust in politicians/experts, media consumption, social media engagement, threat and anxiety, and positive and negative emotions.

\section{Method}

\section{Open science practices.}

The data and stimulus materials can be found on our open science framework page: https://osf.io/sk4dt/

The data analysis (in Python) can be accessed as a jupyter notebook on Github: https://github.com/mvlasceanu/coviddyad All measures, manipulations, and exclusions in this study are disclosed. 


\section{Participants.}

To detect an effect size of 0.5 with $80 \%$ power at a significance level of 0.05 in an independent sample comparison, we recruited a total of 140 participants. Participants were recruited on Amazon Mechanical Turk and were compensated at the platform's standard rate. After discarding participants who failed the pre-established attention checks, the data from the final sample of 126 participants ( $61 \%$ women; Mage $=37.84$, SDage $=11.35$ ) was included in our statistical analyses. The study was approved by the Institutional Review Board at Princeton University.

\section{Stimulus materials.}

We undertook preliminary studies to develop a set of 22 statements regarding COVID19. A pilot study was conducted on separate sample of 269 Cloud Research workers $\left(\mathrm{M}_{\mathrm{age}}=40.63, \mathrm{SD}_{\mathrm{age}}=15.49 ; 66 \%\right.$ women $)$ to select these statements from a larger initial set of 37 statements. For each of these statements we collected believability ratings (i.e., "How accurate or inaccurate do you think this statement is?" on a scale from 0-Extremely Inaccurate to 100-Extremely Accurate). The 22 statements we selected were on average moderately endorsed ( $\mathrm{M}=51.95, \mathrm{SD}=20.08$, on a 0 to 100-point scale). In reality, 9 of them are actually accurate (e.g., "The sudden loss of smell or taste is a symptom of being infected with COVID-19"), 9 are inaccurate (e.g., "Antibiotics can kill COVID-19"), and 4 are conspiracies (e.g., "COVID-19 was built as an intended bioweapon"), as concluded by 
published scientific papers and/or by the CDC. Of note, conspiracies differ from inaccurate statements by assuming nefarious intent and being immune to evidence (Lewandowsky \& Cook, 2020).

\section{Design and procedure.}

The data was collected in May 2020. The 126 participants went through five experimental phases. Participants were told they would participate in an experiment about people's evaluation of information and were directed to the survey on SoPHIE (i.e., Software Platform for Human Interaction Experiments) a platform that allows freeflowing computer-mediated interactions among participants. After completing the informed consent form, participants were directed to a pre-evaluation phase, in which they rated a set of 22 statements (one on each page) by indicating the degree to which they believed each statement (i.e., "How accurate do you think this statement is," from 1Extremely inaccurate to 10-Extremely accurate). Then, in the pre-emotions phase, participants were asked to fill a series of emotion scales (see Measures). A conversational phase followed, in which participants were randomly paired in groups of two and were instructed to discuss the information from the pre-test phase with another participant, in a 5-minute dyadic conversation. The instructions encouraged a random subset of the pairs to discuss any piece of information from the study ( $N=58$; Low Epistemic Condition): "In what follows you will have a chat conversation with another participant who 
answered the same questions about COVID-19 like yourself. In this conversation, please discuss the information about COVID-19 we asked about at the beginning of this study. As you mention a piece of information please be as specific as possible so that your conversational partner can identify what information you are referring to." The other subset of the pairs were asked to discuss only the pieces of information they were confident were correct $(\mathrm{N}=68$; High Epistemic Condition): "In what follows you will have a chat conversation with another participant who answered the same questions about COVID-19 like yourself. In this conversation, please discuss the information about COVID-19 we asked about at the beginning of this study. Importantly, only discuss information you believe is true and correct the other participant if they bring up information you believe is false. As you mention a piece of information please be as specific as possible so that your conversational partner can identify what information you are referring to." Conversations took the form of interactive exchanges in a chat-like computer-mediated environment in which participants typed their responses. In the next phase (post-test), participants rated again the believability of the initial 22 statements. Finally, in the post-emotions phase, participants rated their emotions again, after which they were asked to complete a demographic questionnaire and were debriefed. 


\section{Measures.}

Statement endorsement was measured at pre-test and post-test with the question "How accurate or inaccurate do you think this statement is?", on a scale from 0-Extremely Inaccurate to 10-Extremely Accurate.

The emotion scales included COVID-19 anxiety, measured in the pre-emotion and post-emotion phase with the question "How anxious are you about the COVID-19 pandemic?" on a scale from 0-Not at all to 100-Extremely.

Another emotion scale was a shorter version of the Positive and Negative Affect Schedule (PANAS; Watson, Clark, Tellegen, 1988), in which we included 8 emotions: Calm, Tense, Relaxed, Worried, Content, Fearful, Hopeful, Anxious, Lonely. The instructions were: "Read each statement and select the appropriate response to indicate how you feel right now, that is, at this moment. There are no right or wrong answers. Do not spend too much time on any one statement and give the answer which seems to describe your present feelings best" and participants rated each emotion from 0-Not at all to 5-Extremely. We included the PANAS both in the pre-emotion phase and in the post-emotion phase. In our analyses, we aggregated the 4 positive emotions and the 5 negative emotions to create a measure of PANAS positive emotions and one of PANAS negative emotions.

Moreover, dynamic anxiety was measured by the question "Would you say that during the past 6 weeks you have become more or less anxious about the COVID-19 pandemic?" 
on a scale from 1-Much less anxious to 7-Much more anxious. We only measured dynamic anxiety in the pre-emotions phase.

The final emotion we measured was COVID-19 threat, with the question "How threatening is the COVID-19 pandemic?" from 0-Not at all to 10-Extremely.

Participants' news media and social media usage was measured as part of the demographic section at the end of the experiment. Engagement with news media was measured with the question "During a regular day the last 2 weeks, how many hours a day have you been watching the following media outlets (approximate to whole number)" on a scale from 0 ( 0 hours) to 5 ( 5 or more hours). We included the following media outlets: MSNBC, $C N N, F O X, A B C, N B C, C B S$, and $P B S / N P R$. In our analyses, we used both the individual media outlet data as well as the aggregated score of all 7 media outlets. The aggregated score consisted of the sum of all the answers on each of the 7 scales, creating a single news media measure.

Similarly, for social media, we asked participants "During a regular day the last 2 weeks, how many minutes a day have you been on the following social media platforms (approximate to whole number)" on a scale from 0 (0 minutes) to 10 (100 minutes or more). The social media platforms we included were Facebook, Instagram, Twitter, and Snapchat. We aggregated these 4 social media platforms in our analyses by summing all the answers on each of these scales to create a single social media measure. 
Also in the demographic section we measured participants' trust in President Trump with the question "How much do you trust the COVID-19 information provided by President Trump?" and trust in Dr. Fauci with the question "How much do you trust the COVID-19 information provided by Doctor Anthony Fauci, the director of the National Institute of Allergy and Infectious Diseases?" on a scale from 0-Not at all to 10-Extremely.

Finally, we asked participants to indicate their age, gender, education, and political orientation.

\section{Analysis and coding.}

Participants' knowledge about COVID-19 was computed as the difference between their endorsement of the accurate and the inaccurate information. Participants' COVID-19 conspiracy beliefs were analyzed separately. Together, knowledge (belief in accurate minus inaccurate information) and conspiracies are referred to as beliefs. Belief change was computed as participants' statement endorsement at post-test minus endorsement at pre-test. Therefore, we note that even though participants scored their beliefs on a 010 scale, a belief change score could go from -10 to 10.

The conversations' content was coded for belief endorsement. This entailed marking the statements that were endorsed or refuted in conversation, or simply not brought up at all. We used a coding rubric by which a mentioned statement was labeled as either strongly endorsed (+3), endorsed (+2), slightly endorsed (+1), not mentioned 
(0), slightly opposed (-1), opposed (-2), or strongly opposed (-3). For example, the phrase "Antibiotics can kill COVID-19" would be assigned a +1 , the phrase "I really think that antibiotics can kill COVID-19" would get a +2 , and the phrase "I really think that antibiotics can kill COVID-19 because I've read studies that say it." a +3. Conversely, the coding scheme was the mirror opposite for the opposing statements. For each participant we accounted for both their own input (i.e., self-endorsement, coded from -3 to +3 ) as well as their conversational partner's input (i.e., partner-endorsement, coded from -3 to +3 ) for each statement. Ten percent of the data were double coded for reliability (Cohen $\kappa>0.88$ ), and all disagreements were resolved through discussion between coders.

\section{Results}

\section{Low versus High Epistemic Conditions.}

To test our first hypothesis, that participants in the high epistemic condition would increase more in knowledge compared to those in the low epistemic condition, we ran a repeated measures ANOVA nested by conversational dyads to account for participants' interactions in the conversational phase. We included belief change as the dependent variable, epistemic condition (low and high epistemic) as the between-subject variable, and belief type (accurate, inaccurate, conspiracy) as the within-subject variable. We found a non-significant main effect of belief type $F(2,114)=0.761, p=0.470, \eta p 2=0.013$, a 
non-significant main effect of condition, $\mathrm{F}(1,57)=0.151, \mathrm{p}=0.699$, $\eta \mathrm{p} 2=0.003$, and a nonsignificant interaction $\mathrm{F}(2,114)=0.015, \mathrm{p}=0.955, \eta \mathrm{p} 2=0.001$ (Fig. S1). Surprisingly, we found that participants did not change their beliefs differently in the two epistemic conditions: participants in the high epistemic condition did not increase more in knowledge than participants in the low epistemic condition.

Next, we wanted to investigate whether the epistemic manipulation impacted the conversational content. We ran a repeated measures ANOVA nested by conversational dyads, with the epistemic condition (low and high epistemic) as the between-subject variable and belief type (accurate, inaccurate, conspiracy) as the within-subject variable. This time, the dependent variable was the conversational belief endorsement score. We found a significant main effect of belief type $F(2,114)=20.58$, $\mathrm{p}<0.001, \eta p^{2}=0.265$, not of condition, $\mathrm{F}(1,57)=1.00, \mathrm{p}=0.321, \eta^{2}=0.017$, and a significant interaction $\mathrm{F}(2,114)=7.87, \mathrm{p}<0.001, \eta_{p}{ }^{2}=0.121$. Post-hoc analyses revealed that accurate statements were endorsed more in conversations in the high $(\mathrm{M}=4.05, \mathrm{SD}=4.27)$ than in the low epistemic condition $(\mathrm{M}=1.23, \mathrm{SD}=1.84), \mathrm{t}(74)=4.66, \mathrm{p}<0.001$, Cohen's $\mathrm{d}=0.88, \mathrm{CI}$ $[1.67,3.95]$. However, inaccurate statements were similarly endorsed in the high $(\mathrm{M}=-$ $0.84, \mathrm{SD}=3.18)$ and low $(\mathrm{M}=-0.11, \mathrm{SD}=0.82)$ epistemic conditions $(\mathrm{p}=0.09)$, and conspiracies were also similarly endorsed in the high $(\mathrm{M}=-0.48, \mathrm{SD}=2.17)$ and low $(\mathrm{M}=0.22, \mathrm{SD}=3.62)$ epistemic conditions $(\mathrm{p}=0.18)$. Therefore, we found that, as intended, 
participants endorsed more accurate statements in their conversations in the high epistemic condition (Fig. 1).
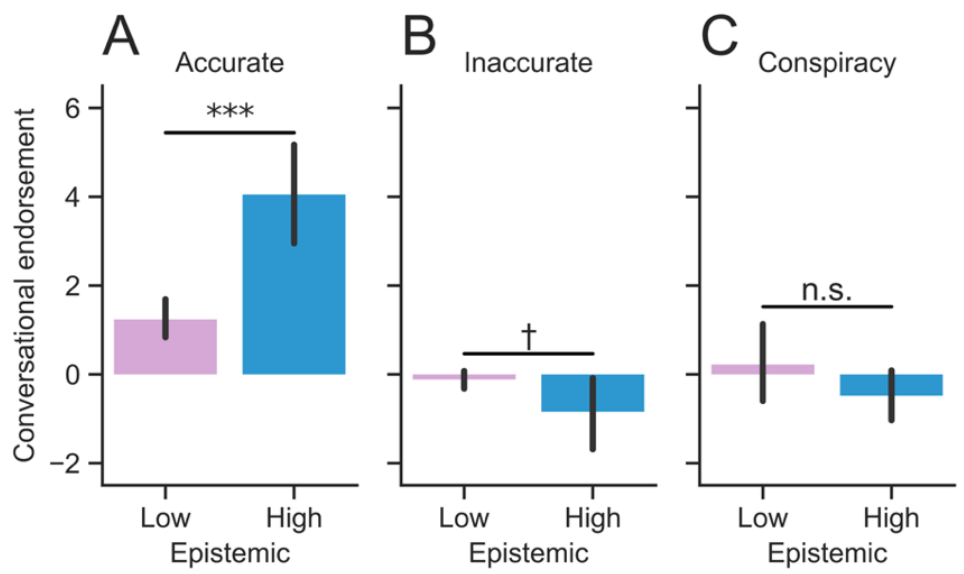

Figure 1. Conversational statement endorsement (joint self and partner) in the Low (Pink) and High (Blue) Epistemic Conditions, split by statement type: Accurate (Panel A), Inaccurate (Panel B) and Conspiracy (Panel C). Note that because on the y axis we plot the joint endorsement, the interval is $[-6,6]$. Error bars represent \pm 1 standard errors of the mean.

\section{Belief change as a function of conversational interactions.}

To investigate our second hypothesis, that participants would change their beliefs to align with their conversational partner, we ran a linear mixed model with belief change as the dependent variable, partner conversational endorsement as the fixed effect, and by-participant, by-dyad, and by-item random intercepts. We found that indeed, partners' conversational belief endorsement triggered participants' belief change 
$(\beta=0.25, \mathrm{SE}=0.06, \mathrm{t}(2402)=4.00, \mathrm{p}<0.001)$. This relationship remained significant $(\beta=0.22$,

$\mathrm{SE}=0.06, \mathrm{t}(2545)=3.33, \mathrm{p}<0.001)$ even when controlling for participants' own

conversational belief endorsement, by including self-conversational belief endorsement

as another fixed effect in the model. Therefore, participants were sensitive to their

conversational partners' endorsement of the statements, and changed their beliefs

accordingly, such that the more their partner expressed disagreement with a statement in conversation the more the participant decreased their belief in that statement, and the more their partner expressed agreement with a statement in conversation, the more the participant increased their belief in that statement (Fig. 2A). This effect did not interact significantly with the Epistemic Condition (see Supplementary Materials).
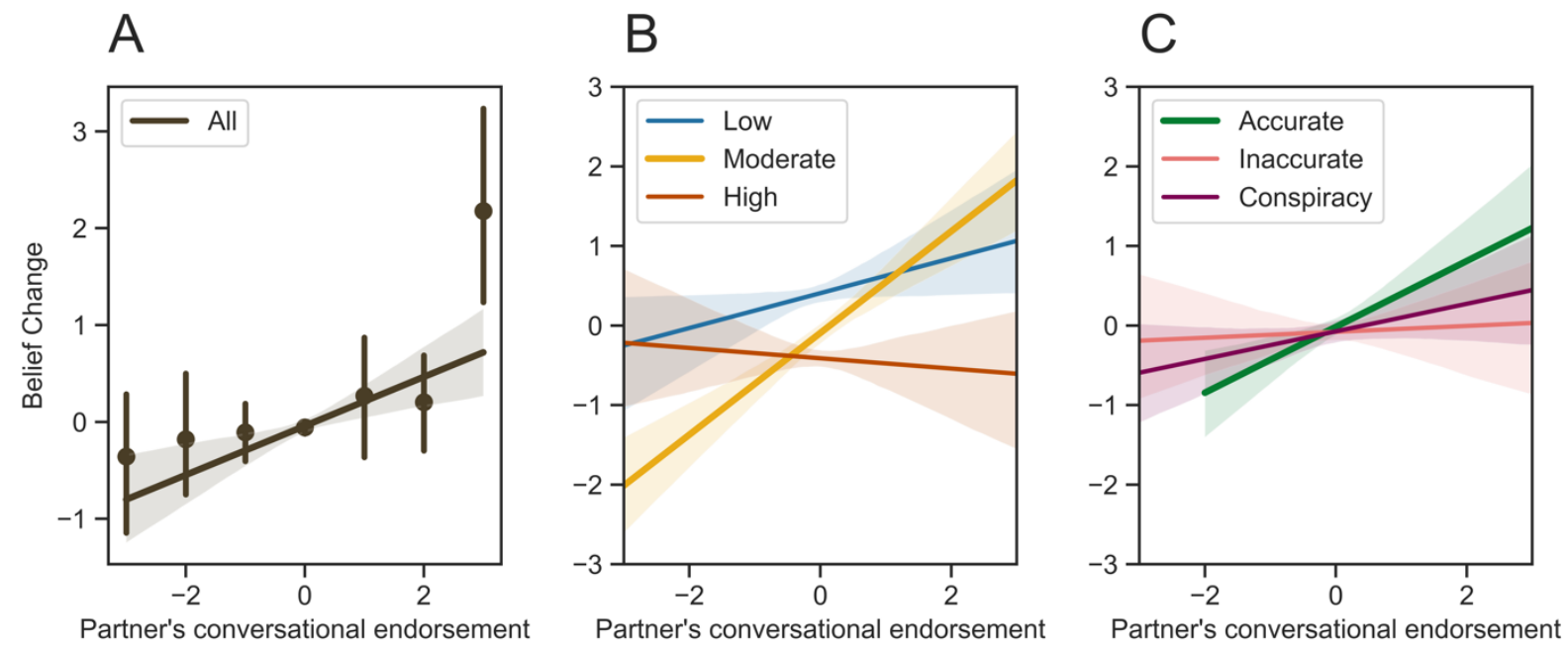

Figure 2. Belief update (self) as a function of the conversational partner's belief endorsement as conveyed in the conversational interaction, for all statements (Panel A), for the statements split into Low (blue), Moderate (yellow), and High (orange) endorsed 
statements at pre-test (Panel B), and for statements split into Accurate (Green), Inaccurate (Red), and Conspiracy (Purple) statements. Error bars represent 95\% bootstrapped confidence intervals on the means.

Furthermore, to uncover which beliefs were most susceptible to change, we split the 22 statements into 3 categories for each participant, according to their pre-test ratings, as: low endorsement (lowest rated 7 statements), moderate endorsement (middle 8 statements), and high endorsement (highest 7 statements). We then ran a linear mixed model with belief change as the dependent variable, partner conversational endorsement and type (low, mod, high) as fixed effects, with byparticipant, by-dyad, and by-item random intercepts. We found that partner conversational endorsement significantly triggered belief change for the initially moderately endorsed statements $(\beta=0.60, \mathrm{SE}=0.09, \mathrm{t}(2578)=6.35, \mathrm{p}<0.001)$, but not for the initially low $(\beta=0.19, \mathrm{SE}=0.12, \mathrm{t}(2582)=1.51, \mathrm{p}=0.133)$ or high $(\beta=-0.19, \mathrm{SE}=0.11, \mathrm{t}(2415)=-$ 1.69, $\mathrm{p}=0.090$ ) endorsed statements. Therefore, as hypothesized, participants' sensitivity to their conversational partners' statement endorsement was driven by the beliefs they initially moderately endorsed (Fig. 2B).

Lastly, to further explore which statements were most susceptible to change, we split the 22 statements by their actual accuracy, into accurate ( 9 statements), inaccurate (9 statements), and conspiracy (4 statements). To investigate whether item type as a 
function of partner conversational endorsement rendered any differences in belief change, we ran a linear mixed model, with belief change as the dependent variable, partner conversational endorsement and type (accurate, inaccurate, conspiracy) as fixed effects, and by-participant, by-dyad, and by-item random intercepts. We found that partner conversational endorsement significantly triggered belief change for the accurate statements $(\beta=0.42, \mathrm{SE}=0.09, \mathrm{t}(1862)=4.39, \mathrm{p}<0.001)$, but not for the inaccurate $(\beta=0.04, \mathrm{SE}=0.12, \mathrm{t}(2581)=0.31, \mathrm{p}=0.752)$ or the conspiracy statements $(\beta=0.19, \mathrm{SE}=0.11$, $\mathrm{t}(2588)=1.70, \mathrm{p}=0.088)$. Therefore, participants' sensitivity to their conversational partners' statement endorsement was driven by the accurate statements (Fig. 2C).

\section{Exploratory analyses.}

In exploratory analyses, we first tested which variables predicted knowledge, and which predicted believing conspiracies. For knowledge (i.e., belief in accurate information minus belief in inaccurate information), we ran a linear mixed model with knowledge at pre-test as the dependent variable; the fixed effect variables we included were: education level, age, gender, political orientation, trust in Trump, trust in Fauci, news media, social media, COVID-19 threat, COVID-19 anxiety, dynamic anxiety, PANAS positive emotions, PANAS negative emotions. Of these, the significant predictors of knowledge were not trusting Trump, trusting Fauci, and COVID-19 threat (Table 1). 


\begin{tabular}{lllllll} 
& $\beta$ & $S E$ & $d f$ & $t$ & $p$ & \\
\hline (Intercept) & 3.06 & 1.31 & 112 & 2.32 & 0.021 & $*$ \\
Trust in Trump & -0.13 & 0.06 & 112 & -2.02 & 0.045 & $*$ \\
Trust in Fauci & 0.21 & 0.08 & 112 & 2.64 & 0.009 & $* *$ \\
COVID-19 threat & 0.18 & 0.07 & 112 & 2.35 & 0.020 & $*$ \\
COVID-19 anxiety & -0.02 & 0.08 & 112 & -0.27 & 0.784 & \\
Dynamic anxiety & -0.11 & 0.13 & 112 & -0.85 & 0.394 & \\
PANAS positive & -0.31 & 0.20 & 112 & -1.54 & 0.125 & \\
PANAS negative & -0.35 & 0.24 & 112 & -1.45 & 0.149 & \\
News media & -0.04 & 0.03 & 112 & -1.34 & 0.182 & \\
Social media & -0.01 & 0.02 & 112 & -0.43 & 0.662 & \\
Age & 0.02 & 0.01 & 112 & 1.77 & 0.077 & \\
Education & 0.03 & 0.17 & 112 & -0.20 & 0.837 & \\
Gender (F) & 0.05 & 0.33 & 112 & 0.17 & 0.858 & \\
Political orientation (D) & -0.01 & 0.41 & 112 & -0.02 & 0.981 & \\
Political orientation (R) & -0.16 & 0.55 & 112 & -0.30 & 0.760 &
\end{tabular}

Table 1. Linear mixed model predicting knowledge at pre-test.

For conspiracy beliefs, we ran the same linear mixed model, except the dependent variable was conspiracy endorsement at pre-test. The significant predictors of believing conspiracies were trusting Trump, not trusting Fauci, news media consumption, social media engagement, and political orientation (Table 2).

\begin{tabular}{lllllll} 
& $\beta$ & $S E$ & $d f$ & $t$ & $p$ & \\
\hline (Intercept) & 1.79 & 1.19 & 112 & 1.50 & 0.135 & \\
Trust in Trump & 0.21 & 0.05 & 112 & 3.68 & $<0.001$ & $* * *$ \\
Trust in Fauci & -0.28 & 0.07 & 112 & -3.81 & $<0.001$ & $* * *$ \\
COVID-19 threat & 0.09 & 0.06 & 112 & 1.34 & 0.180 & \\
COVID-19 anxiety & 0.02 & 0.07 & 112 & 0.33 & 0.740 & \\
Dynamic anxiety & -0.07 & 0.12 & 112 & -0.65 & 0.517 & \\
PANAS positive & 0.22 & 0.18 & 112 & 1.20 & 0.229 & \\
PANAS negative & 0.14 & 0.21 & 112 & 0.67 & 0.498 & \multirow{2}{*}{ New } \\
News media & 0.11 & 0.03 & 112 & 3.68 & $<0.001$ & $* * *$ \\
Social media & 0.04 & 0.02 & 112 & 2.01 & 0.046 & $*$
\end{tabular}




$\begin{array}{llllllll}\text { Age } & 0.004 & 0.01 & 112 & 0.31 & 0.755 & \\ \text { Education } & -0.11 & 0.16 & 112 & -0.70 & 0.482 & \\ \text { Gender (F) } & -0.43 & 0.29 & 112 & -1.45 & 0.149 & \\ \text { Political orientation (D) } & -0.77 & 0.37 & 112 & -2.08 & 0.039 & * \\ \text { Political orientation (R) } & 0.01 & 0.49 & 112 & 0.02 & 0.982 & \end{array}$

Table 2. Linear mixed model predicting conspiracy at pre-test.

To more intuitively display the significant predictors of knowledge and conspiracy beliefs in the two mixed models above, we plotted the regressions of trust in Trump and trust in Fauci (Fig. 3) as well as news media consumption and social media participation (Fig. 4), against knowledge and conspiracy belief.
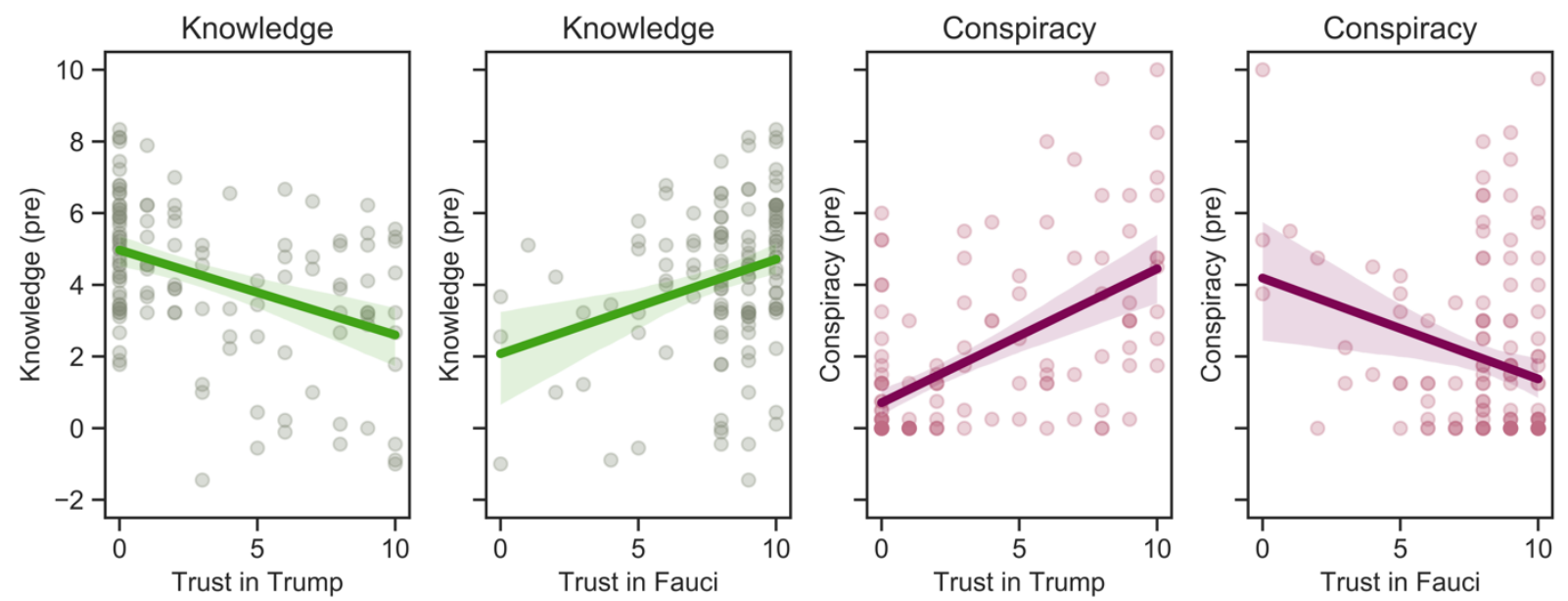

Figure 3. Knowledge (Green) and Conspiracy (Purple) at pre-test, as a function of trust in Trump and trust in Fauci. 

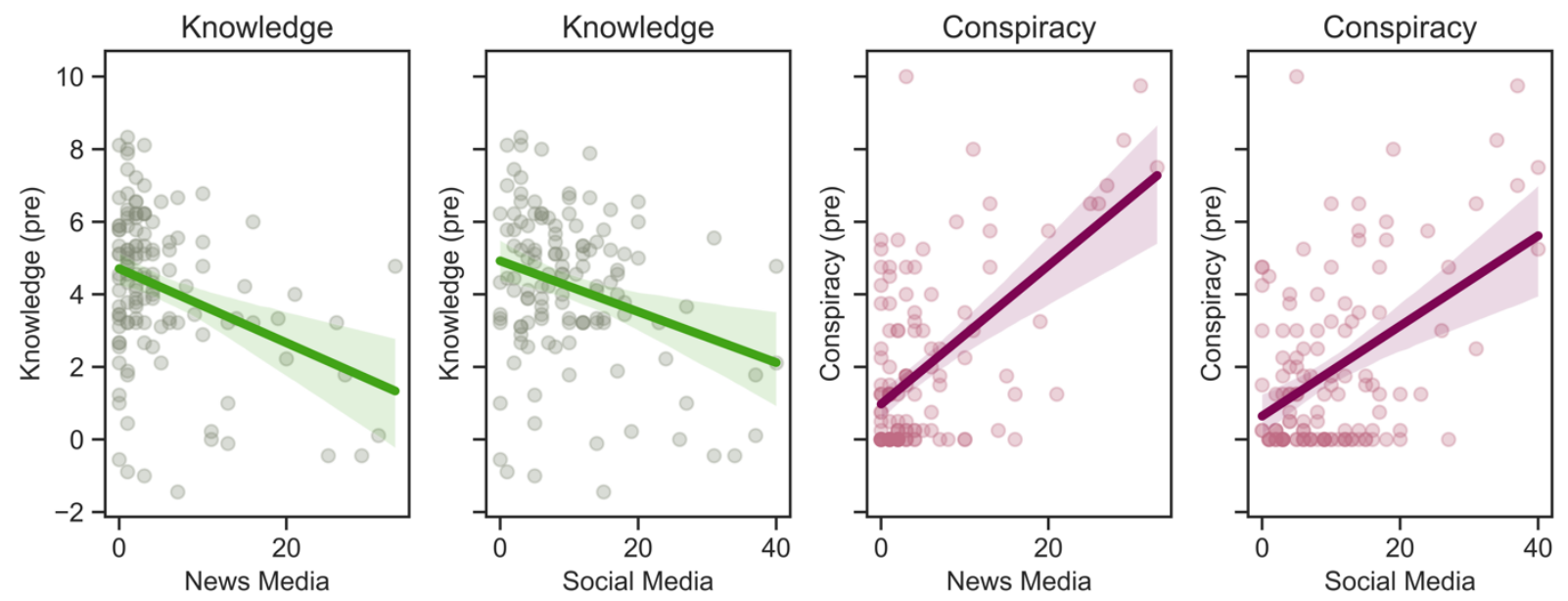

Figure 4. Knowledge (Green) and Conspiracy (Purple) at pre-test, as a function of News Media consumption and Social Media usage.

Given the surprising result that news media consumption predicted conspiracy beliefs, we wanted to investigate whether this effect was driven by a particular news source, or whether it was a general effect of all news networks. We ran a linear mixed model with conspiracy endorsement at pre-test as the dependent variable, news media consumption and network (FOX, CNN, ABC, MSNBC, NBC, CBS, PBS) as fixed effects, and by-participant random intercepts. We found no interaction of news consumption with news network $(\mathrm{p}=0.9)$, suggesting that news consumption of any of the 7 networks predicted conspiracy beliefs (Fig. 5). For the sake of completion, even though not significant, we also plotted the news media consumption of each network as it predicted knowledge (Fig. 5). 
For additional ideological differences found in our sample, please refer to Supplementary Materials.
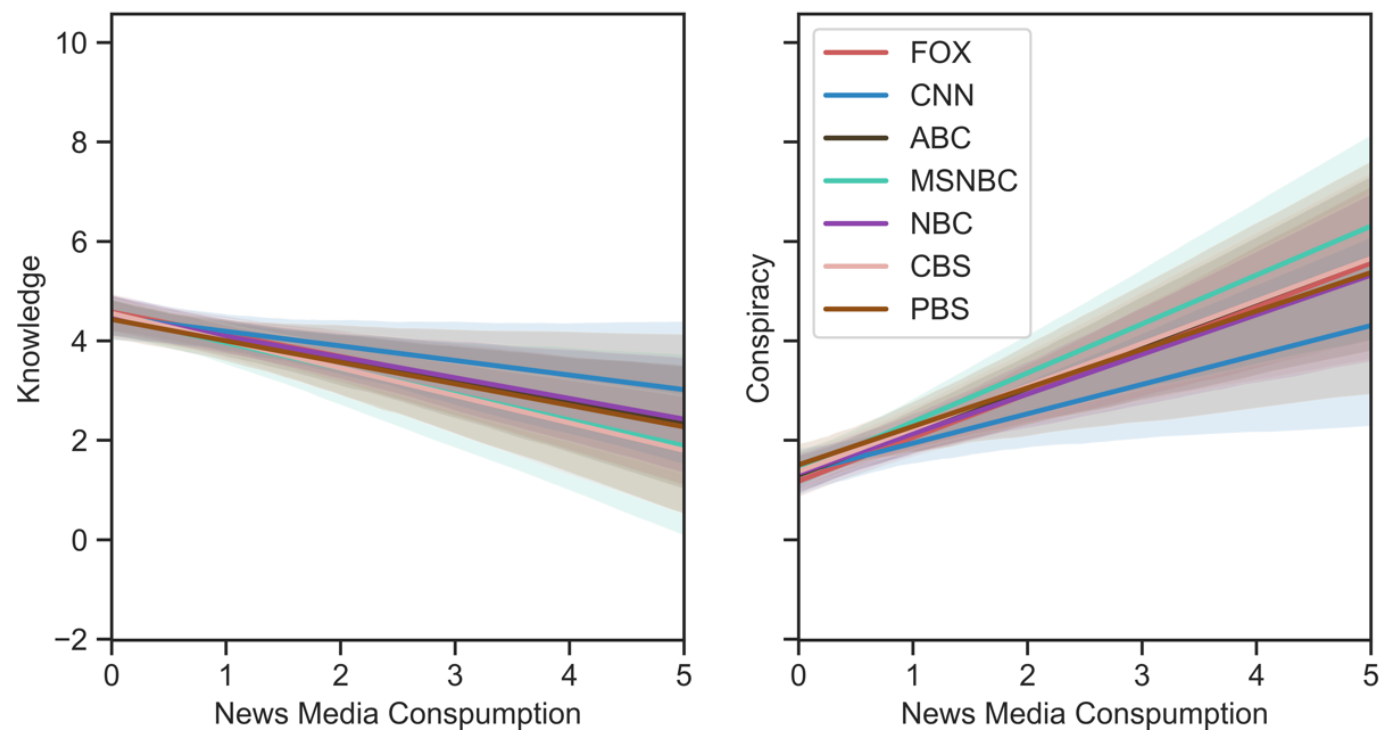

Figure 5. Knowledge (left) and Conspiracy (right) at pre-test, as a function of News Media consumption, by news networks.

\section{Discussion}

In a high-risk environment, such as during an epidemic, people are exposed to a large amount of information, both accurate and inaccurate, which they typically discuss with each other in conversations. Here, we investigated the effectiveness of deploying a high epistemic accuracy manipulation on people's free-flowing communicative interactions regarding COVID-19, and their knowledge accumulation as a result of these interactions. In line to prior research showing the benefits of nudging epistemic 
accuracy, such as sharing less misinformation (Lewandowsky et al., 2012; Pennycook et al., 2021), we found that participants in the high versus low epistemic condition discussed more accurate information. However, in contrast to these prior studies, we found that the difference in conversational content did not lead to differences in how knowledgeable participants in the two conditions became as a result of conversations. This finding points to a higher resistance to this manipulation when it comes to changing one's beliefs compared to simply choosing what to discuss and propagate. Therefore, this study provides evidence for a possible boundary condition of these types of interventions.

We speculate that one reason the epistemic accuracy manipulation did not have the intended effect could be that while the belief change was computed for all the statements tested, only a small subset of them (16\%) were on average discussed in each conversation (Figure S3). Talking about these statements did increase participants' postconversational endorsement, but they were too few to impact the entire knowledge score. This observation could open up interesting theoretical avenues. For instance, the size of the set of beliefs one measures can moderate the strength of the epistemic manipulation, such that when few beliefs are measured the epistemic accuracy effect is apparent, whereas when measuring a larger set, it is not.

We also show that, during the coronavirus pandemic, individuals talking to each other are sensitive to their conversational partners, by changing their beliefs regarding 
COVID-19 information according to their partners' conveyed beliefs. This influence is strongest for initially moderately held beliefs (compared to initially endorsed or opposed beliefs), and for accurate information (compared to inaccurate or conspiracy information). This finding extends prior research showing this effect at the level of memory (Cuc et al., 2007), and aligns with prior research showing individuals synchronize their beliefs after engaging in conversations with other participants in social networks (Vlasceanu, Morais, Duker, \& Coman, 2020; Vlasceanu \& Coman, 2020). Lastly, in exploratory analyses, we found that having COVID-19 knowledge is predicted by trusting Fauci, not trusting Trump, and feeling threatened by COVID-19. Conversely, endorsing conspiracies is predicted by trusting Trump, not trusting Fauci, news media consumption, social media usage, and political orientation. These findings, although in need of confirmation through subsequent replications, also align with prior work and have important implications in the current socio-political context. The interaction between ideology and conspiracy endorsement is consistent with prior instances in which Republicans endorsed conspiracy theories more than Democrats (Pasek, Stark, Krosnick, \& Tompson, 2015), and with the general trend in the wider political literature of Republicans being more likely to believe conspiracies about Democrats and vice versa (Hollander, 2018; Smallpage, Enders, \& Uscinski, 2017; Miller, Saunders, \& Farhart, 2016; Oliver \& Wood, 2014; Radnitz \& Underwood, 2015). These trends are applicable in the case of COVID-19, which was labeled a "hoax" by President 
Trump, and a "Democratic hoax" by Eric Trump. A surprising finding was that news media consumption positively predicted believing conspiracies regarding COVID-19, even when controlling for demographic variables such as age, gender, education, and political orientation. This effect was not driven by a particular news network, instead it was a general effect of news media consumption. This finding counters prior work suggesting that people consuming news media are less likely to believe conspiracies (Hollander, 2018; Stempel, Hargrove, \& Stempel III, 2007) and that people who are more knowledgeable about news media are also less likely to endorse conspiracy theories (Craft, Ashley, \& Maksl, 2017). Thus, clarifying the mechanism of this discrepancy would be a worthwhile future trajectory.

Several other research trajectories emerge from this work. For instance, an important aspect of belief change that was omitted here is source credibility (Chung, Fink, \& Kaplowitz, 2008; Slater \& Rouner, 1996; Vlasceanu \& Coman, 2020). This line of work would benefit from future investigations into how the source presenting information might influence the conversational content and belief change, and how this influence might be amplified or attenuated by conversations. Also, future work could investigate whether revealing features of the conversational partner, such as their ideological orientation, might moderate individuals' willingness to change their beliefs as a function of their conversations. The present work could also be extended from the dyadic level to the collective belief level (Vlasceanu, Enz, \& Coman, 2018; Vlasceanu \& 
Coman, 2020) by investigating the effect of multiple conversations within communities on belief change. Critically, these dyadic level influences (i.e., from speaker to listener) have been found to propagate in social networks (Coman, Momennejad, Duker, \& Geana, 2016; Vlasceanu et al., 2020). In line with existing research, it is likely that the high perceived risk of infection might influence the propagation of information through social networks. Tracking information propagation in fully mapped social networks would be critically important, especially given policymakers' interests in impacting communities at scale (Dovidio \& Esses, 2007). 


\section{References}

Berger, J., \& Milkman, K. L. (2012). What makes online content viral?. Journal of marketing research, 49(2), 192-205.

Blumen, H. M., \& Rajaram, S. (2008). Influence of re-exposure and retrieval disruption during group collaboration on later individual recall. Memory, 16(3), 231-244.

Brandt MJ, Sleegers WWA. (2021). Evaluating Belief System Networks as a Theory of Political Belief System Dynamics. Pers Soc Psychol Rev. doi: 10.1177/1088868321993751.

Chung, S., Fink, E. L., \& Kaplowitz, S. A. (2008). The comparative statics and dynamics of beliefs: The effect of message discrepancy and source credibility. Communication Monographs, 75(2), 158-189.

Coman, A., \& Berry, J. N. (2015). Infectious cognition: Risk perception affects socially shared retrieval-induced forgetting of medical information. Psychological science, 26(12), 19651971.

Coman, A., Momennejad, I., Drach, R. D., \& Geana, A. (2016). Mnemonic convergence in social networks: The emergent properties of cognition at a collective level. Proceedings of the National Academy of Sciences, 113(29), 8171-8176.

Craft, S., Ashley, S., \& Maksl, A. (2017). News media literacy and conspiracy theory endorsement. Communication and the Public, 2(4), 388-401.

Cuc, A., Koppel, J., \& Hirst, W. (2007). Silence is not golden: A case for socially shared retrievalinduced forgetting. Psychological Science, 18(8), 727-733.

Dovidio, J. F., \& Esses, V. M. (2007). Psychological research and public policy: Bridging the gap. Social Issues and Policy Review, 1, 5-14. 
Echterhoff, G., Higgins, E. T., \& Groll, S. (2005). Audience-tuning effects on memory: the role of shared reality. Journal of personality and social psychology, 89(3), 257.

Fazio, L. K., Brashier, N. M., Payne, B. K., \& Marsh, E. J. (2015). Knowledge does not protect against illusory truth. Journal of Experimental Psychology: General, 144(5), 993.

Frenkel, S., Alba, D., \& Zhong, R. (2020). Surge of virus misinformation stumps Facebook and Twitter. The New York Times.

Grant, A. M., \& Hofmann, D. A. (2011). Outsourcing inspiration: The performance effects of ideological messages from leaders and beneficiaries. Organizational Behavior and Human Decision Processes, 116(2), 173-187.

Harber, K. D., \& Cohen, D. J. (2005). The emotional broadcaster theory of social sharing. Journal of Language and Social Psychology, 24(4), 382-400.

Hasher, L., Goldstein, D., \& Toppino, T. (1977). Frequency and the conference of referential validity. Journal of verbal learning and verbal behavior, 16(1), 107-112.

Hirst, W., \& Echterhoff, G. (2012). Remembering in conversations: The social sharing and reshaping of memories. Annual review of psychology, 63, 55-79.

Hollander, B. A. (2018). Partisanship, individual differences, and news media exposure as predictors of conspiracy beliefs. Journalism E Mass Communication Quarterly, 95(3), 691713.

Lewandowsky, S., Ecker, U. K., Seifert, C. M., Schwarz, N., \& Cook, J. (2012). Misinformation and its correction: Continued influence and successful debiasing. Psychological science in the public interest, 13(3), 106-131. 
Lewandowsky, S., \& Cook, J. (2020). The Conspiracy Theory Handbook. Available at http://sks.to/conspiracy

Liu, B. F., Jin, Y., \& Austin, L. L. (2013). The tendency to tell: Understanding publics' communicative responses to crisis information form and source. Journal of Public Relations Research, 25(1), 51-67.

Miller, J. M., Saunders, K. L., \& Farhart, C. E. (2016). Conspiracy endorsement as motivated reasoning: The moderating roles of political knowledge and trust. American Journal of Political Science, 60(4), 824-844.

Nefes, T. S. (2014). Rationale of conspiracy theorizing: Who shot the president Chen Shui-bian? Rationality and Society, 26, 373-394.

Nyhof, M., \& Barrett, J. (2001). Spreading non-natural concepts: The role of intuitive conceptual structures in memory and transmission of cultural materials. Journal of cognition and culture, 1(1), 69-100.

Oliver, J. E., \& Wood, T. (2014). Medical conspiracy theories and health behaviors in the United States. JAMA internal medicine, 174(5), 817-818.

Pasek, J., Stark, T. H., Krosnick, J. A., \& Tompson, T. (2015). What motivates a conspiracy theory? Birther beliefs, partisanship, liberal-conservative ideology, and anti-Black attitudes. Electoral Studies, 40, 482-489.

Pennycook, G., Epstein, Z., Mosleh, M. et al. (2021). Shifting attention to accuracy can reduce misinformation online. Nature. https://doi.org/10.1038/s41586-021-03344-2 
Radnitz, S., \& Underwood, P. (2017). Is belief in conspiracy theories pathological? A survey experiment on the cognitive roots of extreme suspicion. British Journal of Political Science, 47(1), 113-129.

Rozin, P., \& Royzman, E. B. (2001). Negativity bias, negativity dominance, and contagion. Personality and social psychology review, 5(4), 296-320.

Saker, L., Lee, K., Cannito, B., Gilmore, A., \& Campbell-Lendrum, D. H. (2004). Globalization and infectious diseases: a review of the linkages (No. TDR/STR/SEB/ST/04.2). World Health Organization.

Slater, M. D., \& Rouner, D. (1996). How message evaluation and source attributes may influence credibility assessment and belief change. Journalism \& Mass Communication Quarterly, 73(4), 974-991.

Smallpage, S. M., Enders, A. M., \& Uscinski, J. E. (2017). The partisan contours of conspiracy theory beliefs. Research \& Politics, 4(4), 2053168017746554.

Stempel, C., Hargrove, T., \& Stempel III, G. H. (2007). Media use, social structure, and belief in 9/11 conspiracy theories. Journalism \& Mass Communication Quarterly, 84(2), 353-372.

Stojanov A, Bering JM, Halberstadt J. (2020) Does Perceived Lack of Control Lead to Conspiracy Theory Beliefs? Findings from an online MTurk sample. PLoS ONE 15(8): e0237771.

Thiriot, S. (2018). Word-of-mouth dynamics with information seeking: Information is not (only) epidemics. Physica A: Statistical Mechanics and its Applications, 492, 418-430.

Uscinski, J. E., \& Parent, J. M. (2014). American conspiracy theories. Oxford, UK: Oxford University Press. 
Vlasceanu, M., \& Coman, A. (2018). Mnemonic accessibility affects statement believability: The effect of listening to others selectively practicing beliefs. Cognition, 180, 238-245.

Vlasceanu, M., Enz, K., \& Coman, A. (2018). Cognition in a social context: a social-interactionist approach to emergent phenomena. Current Directions in Psychological Science, 27(5), 369377.

Vlasceanu, M., Morais, M.J., Duker, A., \& Coman, A. (2020). The Synchronization of Collective Beliefs: From Dyadic Interactions to Network Convergence. Journal of Experimental Psychology: Applied. Advance online publication. http://dx.doi.org/10.1037/xap0000265

Vlasceanu, M., \& Coman, A. (2020). Network Structure Impacts the Synchronization of Collective Beliefs. https://doi.org/10.31234/osf.io/7rq4g

Vlasceanu, M., \& Coman, A. (2020). Information Sources Differentially Trigger CoronavirusRelated Belief Change. https://doi.org/10.31234/osf.io/5xkst

Whitson, J. A., \& Galinsky, A. D. (2008). Lacking control increases illusory pattern perception. Science, 322(5898), 115-117. 